\title{
Frictionless contact problem between a rigid moving punch and a homogeneous layer resting on a Winkler foundation
}

\author{
İ. Çömez ${ }^{1 *}$, K.B. Yılmaz², M.A. Güler³, B. Yıldırım² \\ ${ }^{1}$ Karadeniz Technical University, Civil Engineering Department, Trabzon, Turkey \\ ${ }^{2}$ Hacettepe University, Department of Mechanical Engineering, Ankara, Turkey \\ ${ }^{3}$ American University of the Middle East, College of Engineering and Technology, Kuwait
}

\begin{abstract}
In this paper, the moving contact problem between the punch and a layer supported by a Winkler type foundation is considered under plane strain conditions. The cylindrical punch moves smoothly with constant velocity over the homogeneous layer. With the use of Galilean transformation and Fourier integral transform, the plane contact problem is converted into a Cauchy type singular integral equation. The singular integral equation is solved numerically by performing the appropriate collocation method, and the contact width, the contact and in-plane stresses are calculated. The results show that the stiffness of the Winkler foundation and the moving velocity have a significant effect on the contact width and the behavior of the stress field.
\end{abstract}

\section{Keywords}

Contact problem; Moving punch; Winkler foundation; Singular integral equation

Received: 18 March 2019; Accepted: 13 June 2019

ISSN: 2630-5763 (online) C 2019 Golden Light Publishing® All rights reserved.

\section{Introduction}

The contact mechanics of deformable components are among the most difficult problems in mechanics. Since mechanical and structural components are usually in contact with each other, contact problems have gained importance over the years. Particularly, the contact problem of a layer resting on an elastic foundation lies at the hearth of soil mechanics.

The studies on contact mechanics have focused on functionally graded materials (FGMs) in recent years. The load transfer problems in the presence of friction (sliding contact) are the potential applications of FGMs [1]. The sliding contact problems of the coated half plane are examined by Guler and Erdogan [1] Guler and Erdogan [2] and Alinia et al. [3] using Fourier integral transform technique. In the mentioned studies, the shear modulus of the coating is assumed to vary exponentially in the depth direction and the system is loaded by rigid cylindrical, flat or triangular stamps. Ke and Wang [4] used the multi-layered model to solve the sliding contact problem of FG coated half plane. With the multi-layered model they divided the FG layer into several sub-layers assuming the elastic properties is a linear function. The sliding contact problem of a half plane coated with a linear graded layer is examined by Chen and Chen [5]. The sliding contact problem of an FG half plane loaded by a flat punch is investigated by Choi [6] and Chen et al. [7]. They also assumed that the shear modulus varies exponentially in an arbitrary direction [7]. The sliding contact analysis of

\footnotetext{
Corresponding author

E-mail: isacomez@ktu.edu.tr
} 
laterally graded medium is investigated by Dag et al. [8] and Dag [9]

In the sliding contact problems, the frictional heating may occur since the presence of friction between the contacting components. The frictional heating may influence the performance of the system and leads to contact damage. Liu et al. [10] examined the thermoelastic contact problem of a FGM coated half plane with multilayered model. Choi and Paulino [11] studied thermoelastic contact of coating-graded interlayer-substrate system. The thermoelastic contact problem of FG coated half plane is studied with finite element method (FEM) by Balci et al. [12]. Barik et al. [13] investigated the effect of thermal conductivity on the thermoelastic contact problem. Mao et al. [14] analyzed the thermoelastic instability of an FGM layer and a homogeneous layer by the perturbation method.

In the absence of body forces the contacting bodies get separated from each other with the loading and the contact area reduces to a finite size. This kind of contact referred to as receding. Rhimi et al. [15] and Rhimi et al. [16] considered the axisymmetric receding contact problem between an FG layer and a homogeneous layer using Hankel transform. The frictional receding contact problems of an FG layer resting on a homogeneous substrate are investigated by El-Borgi et al. [17] and El-Borgi and Çömez [18] using the Fourier transformation technique. The frictionless receding contact problem of a homogeneous layer or bilayer resting on a homogeneous substrate or a layer is examined by Çömez et al. [19], Kahya et al. [20] Adibelli et al. [21]. The layer and substrate are assumed to be anisotropic in [20]. Çömez [22] and Parel and Hills [23] studied the frictional receding contact problem of a homogeneous layer resting on a homogeneous substrate. The studies of Çömez et al. [19] and Adibelli et al. [21] are extended to the FGM case by Jan and Li [24] and Jan and Mi [25].

The contact problem involving a layer supported by a Winkler foundation have many applications particularly in soil mechanics and ice engineering fields. In the Winkler foundation model, it is assumed that the foundation behaves like an infinite number of individual elastic springs and the foundation reaction is proportional to its displacement [26]. Although there are considerable number of studies related to the beam or the plate problem resting on a Winkler foundation, the contact problem of a layer resting on a Winkler foundation is quite limited in the literature. The plane contact problem of an elastic homogeneous layer lying on a Winkler foundation is first investigated by Dempsey et al. [27]. The layer is pressed by line load, uniform load, rigid cylinder or flat punch. This study is extended to the axisymmetric case by Dempsey et al. [28]. Wozniak et al. [29] studied the axisymmetric contact problem of a layer lying on a rigid support with a Winkler type excavitation using the Hankel transform. The contact problem of a homogeneous bilayer supported by a Winkler foundation is analyzed by Birinci et al. [30]. Çömez [26] investigated the contact problem of FG layer supported by Winkler type foundation. Birinci et al. [31] studied the continuous and discontinuous contact problem of the bilayer resting on a Winkler foundation using the FEM. Adryaman and Birinci [32] investigated the contact problem of an FG layer pressed to a Winkler foundation with the arbitrary rigid punches.

There are problems that arise in practice in which the speed of one body relative to other is quite large, and we therefore need to investigate whether it is necessary to take the dynamic character of the problem into account [33]. In this respect, Georgiadis [34] studied the rigid flat moving punch problem over the highly orthotropic strip and examined the effect of the velocity of the punch as well as of the orthotropy. De and Patra [35] investigated the contact problem of a single punch or a row of equally spaced punches moving over the surface of the orthotropic medium. The contact problem between the moving flat or cylindrical punch and the magneto-electro-elastic half plane is analyzed by Zhou and Lee [36], [37]. Zhou et al. [38] and Zhou et al. [39] considered the frictional and frictionless moving contact problem of a rigid punch over the orthotropic layer using Galilean transform and Fourier integral transform technique. It is assumed that the flat or cylindrical 
rigid punch moving at a constant velocity on the surface of orthotropic medium. The first study of the frictionless moving contact problem of an FG layer indented by a rigid cylindrical punch is examined by Çömez [40]. Çömez [41] considered the contact problem between the moving punch and a homogeneous layer in the presence of friction. Balc1 and Dag [42] solved the moving contact problem between the rigid punch and a coated half plane.

It can be seen from the literature that the moving contact problem of a layer resting on a Winkler foundation has not been considered, yet. To fill this gap in the literature, the contact problem of a rigid cylindrical punch moving at a constant velocity on the surface of a homogeneous layer resting on a Winkler foundation is investigated in this study. The plane contact problem was reduced to a singular integral equation of the second kind using the Galilean transformation and Fourier integral transform technique. The integral equation is solved numerically utilizing the Gauss- Chebyshev integration formula. The main objective of this paper is to study effect of the moving velocity and stiffness of the Winkler foundation on the contact area and the distribution of the stress components.

\section{Formulation of the problem}

Consider the plane moving contact problem in Fig 1. A homogeneous layer of a thickness $h$ is resting on a Winkler type foundation. The layer is pressed by a rigid cylindrical punch with radius $R$ subjected to an external force $P$. The punch moves over the surface of the layer in $x_{1}$ direction at a constant velocity $V$.

The governing equations of motion can be written as follows

$$
\begin{aligned}
& \frac{\partial \sigma_{x}}{\partial x_{1}}+\frac{\partial \tau_{x y}}{\partial y_{1}}=\rho \frac{\partial^{2} u}{\partial t^{2}} \\
& \frac{\partial \tau_{y x}}{\partial x_{1}}+\frac{\partial \sigma_{y}}{\partial y_{1}}=\rho \frac{\partial^{2} v}{\partial t^{2}}
\end{aligned}
$$

where $u, v$ are the $x_{1}-$ and $y_{1}-$ components of the displacement vector, respectively and $t$ denotes the time variable.

The stress and displacement relations (Hooke's law) in a state of plane strain can be expressed as follows

$$
\begin{aligned}
& \sigma_{x}=\frac{\mu}{\kappa-1}\left[(\kappa+1) \frac{\partial u}{\partial x_{1}}+(3-\kappa) \frac{\partial v}{\partial y_{1}}\right] \\
& \sigma_{y 1}=\frac{\mu}{\kappa-1}\left[(3-\kappa) \frac{\partial u}{\partial x_{1}}+(\kappa+1) \frac{\partial v}{\partial y_{1}}\right] \\
& \tau_{x y}=\mu\left[\frac{\partial u}{\partial y_{1}}+\frac{\partial v}{\partial x_{1}}\right]
\end{aligned}
$$

where $\mu$ represents the shear modulus and $\kappa=3-4 v$ for the plane strain case, $v$ being the Poisson's ratio.

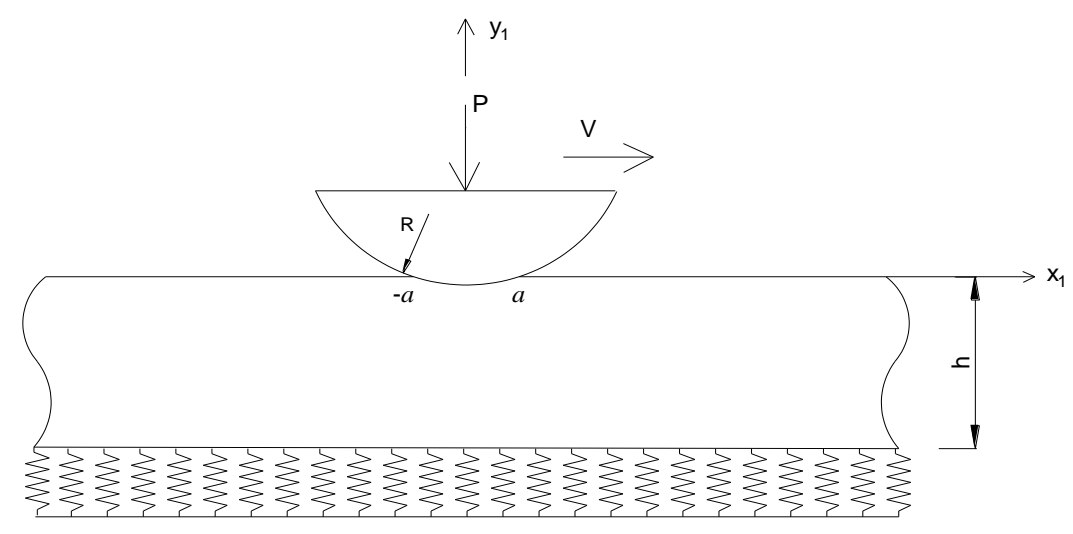

Fig 1. Geometry of the moving contact problem 
Introducing the following Galilean transformation [33], the dynamic contact problem may be turn into the steady contact problem

$$
x=x_{1}-V t \quad y=y_{1}
$$

Substituting Eqs. (3) into Eqs. (1) and using Hooke's law (2), the equations of motion can be written in terms of the derivatives of $u(x, y)$ and $v(x, y)$ as follows

$$
\begin{aligned}
& {\left[(\kappa+1)+(1-\kappa) \frac{V^{2}}{c_{T}^{2}}\right] \frac{\partial^{2} u}{\partial x^{2}}+(\kappa-1) \frac{\partial^{2} u}{\partial y^{2}}+2 \frac{\partial^{2} v}{\partial x \partial y}=0} \\
& -\left[(1-\kappa)+(1+\kappa) \frac{V^{2}}{c_{L}^{2}}\right] \frac{\partial^{2} v}{\partial x^{2}}+(\kappa+1) \frac{\partial^{2} v}{\partial y^{2}}+2 \frac{\partial^{2} u}{\partial x \partial y}=0
\end{aligned}
$$

where $c_{L}$ and $c_{T}$ are the speed of propagation of longitudinal and shear waves in the layer, respectively.

$$
c_{T}=\sqrt{\frac{\mu}{\rho}} \quad c_{L}=\sqrt{\frac{2 \mu+\lambda}{\rho}}
$$

Note that Eqs. (4) contain partial differential equation system. Using the Fourier integral transform technique, Eqs (4) can be converted to the ordinary differential equation system. Due to the plane symmetry, it is adequate to consider the problem in the field $0 \leq x<\infty$ only. Utilizing the symmetry of the problem and Fourier transform technique, the following transforms may be written

$$
\begin{aligned}
& u(x, y)=\frac{2}{\pi} \int_{0}^{\infty} \tilde{u}(\alpha, y) \sin (\alpha x) d \alpha \\
& v(x, y)=\frac{2}{\pi} \int_{0}^{\infty} \tilde{v}(\alpha, y) \cos (\alpha x) d \alpha
\end{aligned}
$$

where $\tilde{u}(\alpha, y)$ and $\tilde{v}(\alpha, y)$ are the Fourier transforms of the displacement vectors $u(x, y)$ and $v(x, y)$, respectively.

Using the Fourier transforms defined (6) to Eqs. (4) with respect to $x$, yields following ordinary differential equation system

$$
\begin{aligned}
& (\kappa-1) \frac{d^{2} \tilde{u}}{d y^{2}}-2 \alpha \frac{d \tilde{v}}{d y}-\alpha^{2}\left[(\kappa+1)+(1-\kappa) \frac{V^{2}}{c_{T}^{2}}\right] \tilde{u}=0 \\
& (\kappa+1) \frac{d^{2} \tilde{v}}{d y^{2}}+2 \alpha \frac{d \tilde{u}}{d y}+\alpha^{2}\left[(1-\kappa)+(1+\kappa) \frac{V^{2}}{c_{L}^{2}}\right] \tilde{v}=0
\end{aligned}
$$

Substituting the necessary derivatives of Eqs. (6) into Eqs. (7) and after some routine manipulations, the following characteristic equation may be obtained

$$
\begin{aligned}
& n^{4}+\left(-2+\frac{V^{2}}{c_{T}^{2}}+\frac{V^{2}}{c_{L}^{2}}\right) n^{2} \\
& +\frac{1}{-1+\kappa^{2}}\left(-1+\frac{V^{2}}{c_{T}^{2}}(-1+\kappa)-\kappa\right) \\
& \times\left(1-\kappa+(1+\kappa) \frac{V^{2}}{c_{L}^{2}}\right)=0
\end{aligned}
$$

The roots of the characteristic equation can be found as

$$
n_{1,2}= \pm \sqrt{1-\frac{V^{2}}{c_{T}^{2}}}, \quad n_{3,4}= \pm \sqrt{1-\frac{V^{2}}{c_{L}^{2}}}
$$

Hence, the solution of the Eqs. (7) may be expressed as

$$
\begin{aligned}
u= & \frac{2}{\pi} \int_{0}^{\infty}\left[A_{1} e^{n_{1} \alpha y}+A_{2} e^{n_{2} \alpha y}+A_{3} e^{-n_{1} \alpha y}\right. \\
& \left.+A_{4} e^{-n_{2} \alpha y}\right] \sin (\alpha x) d \alpha \\
v & =\frac{2}{\pi} \int_{0}^{\infty}\left[-\frac{1}{n_{1}} A_{1} e^{n_{1} \alpha y}-n_{2} A_{2} e^{n_{2} \alpha y}\right. \\
& \left.+\frac{1}{n_{1}} A_{3} e^{-n_{1} \alpha y}+n_{2} A_{4} e^{-n_{2} \alpha y}\right] \cos (\alpha x) d \alpha
\end{aligned}
$$

Using Hooke's law (2) and Eqs. (10) the stress field can be obtained as follows 


$$
\begin{aligned}
\frac{\sigma_{x}}{\mu} & =\frac{2}{\pi} \int_{0}^{\infty} \alpha\left[2 A_{1} e^{n_{1} \alpha y}+\frac{\left(1+n_{2}^{2}(-3+\kappa)+\kappa\right)}{-1+\kappa} A_{2} e^{n_{2} \alpha y}\right. \\
& \left.+2 A_{3} e^{-n_{1} \alpha y}+\frac{\left(1+n_{2}^{2}(-3+\kappa)+\kappa\right)}{-1+\kappa} A_{4} e^{-n_{2} \alpha y}\right]
\end{aligned}
$$$$
\times \cos (\alpha x) d \alpha
$$

$$
\begin{aligned}
\frac{\sigma_{y}}{\mu} & =\frac{2}{\pi} \int_{0}^{\infty}-\alpha\left[2 A_{1} e^{n_{1} \alpha y}+\left(1+n_{1}^{2}\right) A_{2} e^{n_{2} \alpha y}\right. \\
& \left.+2 A_{3} e^{-n_{1} \alpha y}+\left(1+n_{1}^{2}\right) A_{4} e^{-n_{2} \alpha y}\right] \cos (\alpha x) d \alpha
\end{aligned}
$$

$$
\begin{aligned}
\frac{\tau_{x y}}{\mu}= & \frac{2}{\pi} \int_{0}^{\infty} \alpha\left[\frac{\left(1+n_{1}^{2}\right)}{n_{1}} A_{1} e^{n_{1} \alpha y}+2 n_{2} A_{2} e^{n_{2} \alpha y}\right. \\
& \left.-\frac{\left(1+n_{1}^{2}\right)}{n_{1}} A_{3} e^{-n_{1} \alpha y}-2 n_{2} A_{4} e^{-n_{2} \alpha y}\right] \sin (\alpha x) d \alpha
\end{aligned}
$$

where $A_{j}(j=1, \ldots 4)$ are the unknown functions to be determined from the boundary conditions of the problem.

\section{The boundary conditions and the singular integral equation}

The moving contact problem under consideration must be solved using the following boundary conditions

$$
\begin{aligned}
& \sigma_{y}(x, 0)= \begin{cases}-p(x) & 0 \leq x<a \\
0 & a \leq x<\infty\end{cases} \\
& \tau_{x y}(x, 0)=0 \\
& \sigma_{x}(x,-h)-k_{w} v(x,-h)=0 \\
& \tau_{x y}(x,-h)=0
\end{aligned}
$$

where $p(x)$ is the contact stress between the rigid punch and the homogeneous layer on the contact area $(-a, a)$ and $k_{w}$ is the stiffness of the Winkler foundation.

Upon taking the Fourier transforms of the boundary conditions given by (12), the unknowns $A_{j}(j=1, \ldots 4)$ and consequently the displacement and the stress components may be determined in terms of the unknown contact stress $p(x)$. The vertical displacement is known based on the profile of the cylindrical stamp which is cylindrical. Additional condition may be written in the form of a derivative of the rigid punch profile to eliminate rigid-body displacement and ensures continuity of normal displacements as follows

$$
v(x, 0) \cong \frac{x^{2}}{2 R}+\delta, \quad \frac{\partial v(x, 0)}{\partial x}=\frac{x}{R}
$$

where $\delta$ is the maximum indentation depth. Substituting the unknowns $A_{j}$ into the mixed condition (13) and determining the singular behavior of the solution following singular integral equation can be obtained as

$$
\frac{1}{\pi} \int_{-a}^{a} p(\xi) d \xi\left[\frac{1}{\xi-x}+k_{1}(x, \xi)\right]=\frac{\mu}{\varphi_{1}} \frac{x}{R} \quad(0 \leq x<a)
$$

The expressions for $k_{1}(x, \xi)$ and $\varphi_{1}$ from Eq. (14) are given in Appendix A.

In the singular integral equation (14) the contact width $a$ is also unknown, a priori. To complete the solution, $p(x)$ must satisfy the following equilibrium condition

$$
\int_{0}^{\infty} \sigma_{y}(x, 0) d x=-\int_{0}^{a} p(\xi) d \xi=-\frac{P}{2}
$$

\section{Numerical solution of the singular integral equation}

Since the smooth contact at the ends of the contact area, the index of the integral equation (14) is -1 [43].

The following transformations are defined to solve the integral equation numerically

$$
\begin{aligned}
& s=x / a, \quad \xi=r / a \\
& \varphi(r)=\frac{p(r)}{P / h}
\end{aligned}
$$


Using the transformations, the integral equation (14) and the equilibrium condition (15) becomes

$$
\begin{array}{r}
\frac{1}{\pi} \int_{-1}^{1} \phi(r)\left[\frac{1}{r-s}+\frac{a}{h} k_{1}(s, r)\right] d r \\
=\frac{1}{\varphi_{1}} \frac{1}{R / h} \frac{\mu}{P / h} \frac{a}{h} s
\end{array}
$$

$\frac{a}{h} \int_{-1}^{1} \phi(r) d r=1$

The solution of the integral equation can be expressed as

$$
\phi(r)=g(r) \sqrt{\left(1-r^{2}\right)}
$$

where $g(r)$ is a continuous and bonded function in the interval $[-1,1]$. Applying the conventional collocation technique [43], [44] the integral equation (17) can be transformed into the equivalent system of algebraic equations as follows

$$
\begin{aligned}
& \sum_{i=1}^{N} W_{i}^{N}\left[\frac{1}{r_{i}-s_{k}}+\frac{a}{h} k\left(s_{k}, r_{i}\right)\right] g\left(r_{i}\right) \\
& =\frac{1}{\varphi_{1}} \frac{1}{R / h} \frac{\mu}{P / h} \frac{a}{h} s_{k} \quad k=1, \ldots N+1
\end{aligned}
$$

and the equilibrium condition becomes

$$
\frac{a}{h} \sum_{i=1}^{N} W_{i}^{N} g\left(r_{i}\right)=\frac{1}{\pi}
$$

where $r_{i}$ and $s_{k}$ are the roots of the related Chebyshev polynomials and $W_{i}^{N}$ is the weighting constant:

$$
\begin{aligned}
& r_{i}=\cos \left(\frac{i \pi}{N+1}\right) i=1, \ldots N \\
& s_{k}=\cos \left(\frac{\pi}{2} \frac{2 k-1}{N+1}\right) \quad k=1, \ldots N+1 \\
& W_{i}^{N}=\frac{1-r_{i}^{2}}{N+1}
\end{aligned}
$$

Note that there are $N+1$ equations to determine the $N$ unknowns $g\left(r_{i}\right)$ in Eq. 20. It can be shown that the $(\mathrm{N} / 2+1)$-th equation in (20) is automatically satisfied [44]. Thus, Eq (20) and Eq (21) give $N+1$ equations to determine the $N+1$ unknowns, which are $g\left(r_{i}\right)$ and $a$. The system of equations (20) is linear in terms of the $g\left(r_{i}\right)$ but the contact area $a$ is unknown in priory and makes the problem highly nonlinear. Therefore, an iterative method is used to obtain unknowns.

After solving the contact stress and contact areas, the in-plane stress at the surface of the layer can be expressed as

$$
\frac{\sigma_{x}(x, 0)}{P / h}=\left\{\begin{array}{cc}
\varphi_{2} \frac{p(x)}{P / h}+H(x), & -a<x<a \\
H(x), & x \leq-a, x \geq a
\end{array}\right\}
$$

where

$$
\begin{aligned}
& H(x)=\frac{1}{\pi} \int_{-a}^{a} p(\xi) k_{2}(x, \xi) d \xi \\
& k_{2}(x, \xi)=\int_{0}^{\infty}\left(M_{2}(\alpha)-\varphi_{2}\right) \cos \alpha \xi \cos \alpha x d \alpha \\
& M_{2}(\alpha)=\alpha\left[2 A_{1}+\frac{\left(1+n_{2}^{2}(-3+\kappa)+\kappa\right)}{-1+\kappa} A_{2}+2 A_{3}\right. \\
& \left.+\frac{\left(1+n_{2}^{2}(-3+\kappa)+\kappa\right)}{-1+\kappa} A_{4}\right] \\
& \varphi_{2}=\lim _{\alpha \rightarrow \infty} M_{2}(\alpha)
\end{aligned}
$$

\section{Numerical results}

The contact width $a / h$, the contact stress $p(x) /(P / h)$, the in-plane stress component on the surface of the layer $\sigma_{x}(x, 0) /(P / h)$ and along the $\mathrm{y}$ axis $\sigma_{x}(0, y) /(P / h)$ is computed numerically for various material and geometrical parameters such as the moving velocity $V^{2} \rho / \mu$, the stiffness of the Winkler foundation $k_{w} / \mu$, the punch radius $R / h$ and the external load $P /(\mu h)$. During the computation, the Poisson's ratio is taken as $v=0.2$ The results obtained from the study are presented in table and showed graphically in figures. 
This study can be validated with those of Çömez [26] by letting the problem static, i. e. $V^{2} \rho / \mu \rightarrow 0$ For the comparison the contact widths obtained from the two studies are presented in Table 1. It can be observed from the table that the numerical results for the contact width under the punch is quite close to the each other.

Fig 2. shows the influences of the moving velocity $V^{2} \rho / \mu$ and punch radius $R / h$ on the contact width. It may be seen from the figure that an increase in the moving velocity and punch radius tends to widen the contact width. The effect of the moving velocity on the contact width becomes certain when the punch radius gets bigger. Note that when the punch radius very small the contact width does not change with moving velocity.

The effect of the stiffness of the Winkler foundation $k_{w} / \mu$ and the load parameter $P /(\mu h)$ on the contact width is depicted in Fig 3. Note that the foundation becomes flexible when the stiffness of the Winkler foundation is getting smaller. As a result, the contact width tends to widen with decreasing values of the stiffness of the Winkler foundation. When the external load increases, the punch penetrates the layer more, so the contact width increases. It can be observed from the figure that the contact width close to zero when the external load decreases.

Due to the absence of the friction the contact stress and the in-plane stress distributions on the upper surface of the layer are symmetric (Figs. 46). The

Table 1. Comparison of the contact width under the punch with that of Çömez

(2013) $(R / h=100, \mu /(P / h)=500)$

\begin{tabular}{cccc}
\hline \multicolumn{4}{c}{$a / h$} \\
\hline$k_{w} / \mu$ & $\begin{array}{c}\text { Comez, } \\
2013 \\
(\gamma h \cong 0)\end{array}$ & $\begin{array}{c}\text { This study } \\
\left(V^{2} \rho / \mu \cong 0\right)\end{array}$ & $\begin{array}{c}\text { Error } \\
\%\end{array}$ \\
\hline 0.1 & 0.3725 & 0.3729 & $\mathbf{0 . 1 0 7 4}$ \\
1 & 0.3304 & 0.3306 & $\mathbf{0 . 0 6 0 5}$ \\
10 & 0.3151 & 0.3152 & $\mathbf{0 . 0 3 1 7 3}$ \\
$\infty$ & 0.3116 & 0.3117 & $\mathbf{0 . 0 3 2 0 9}$ \\
\hline
\end{tabular}

peak values of the contact stress and the in-plane stress $\sigma_{x}(x, 0) /(P / h)$ occurs at the symmetry plane $x=0$. It is observed from the Figs. (4-6) the tensile peaks occur both on the upper and bottom surface of the layer. The in-plane stress gives some implications for the crack propagation problem. The axial stress $\sigma_{x}(0, y) /(P / h)$ is compressive in the upper part of the layer and tensile in the lower part of the layer.

The influences of the moving velocity on the contact stress and in-plane stresses are plotted in Fig 4. As the punch moves faster the peak values of the contact stress decreases but the tensile peak increase at the bottom and top surface of the layer.

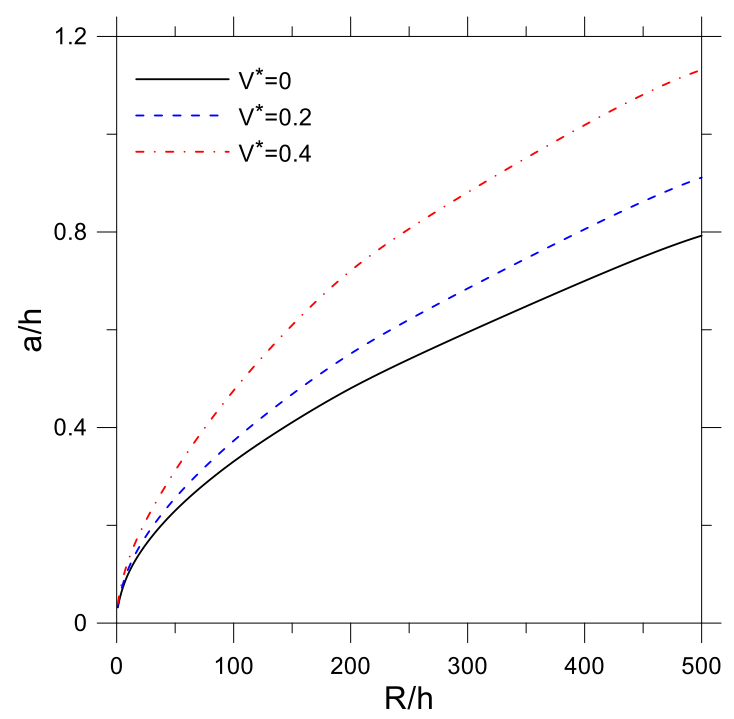

Fig 2. Variations of the contact width under the punch $a / h$ versus punch radius $R / h$ for some selected moving velocity $V^{*}=V^{2} \rho / \mu$ with fixed parameters $k_{w} / \mu=1, \mu /(P / h)=500$. 


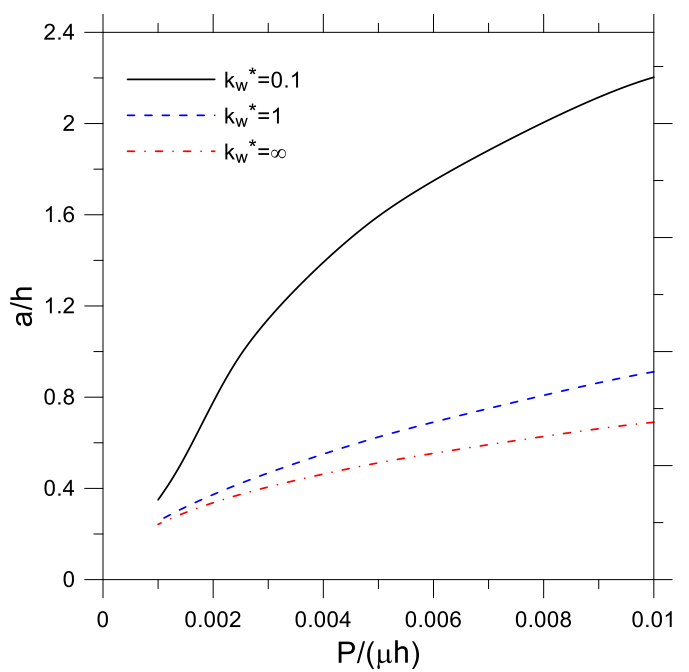

Fig 3. Variations of the contact width under the punch $a / h$ versus load parameter $P /(\mu h)$ for some selected the stiffness of the Winkler foundation $k_{w}^{*}=k_{w} / \mu$ with fixed parameters $V^{2} \rho / \mu=0.2, R / h=100$.

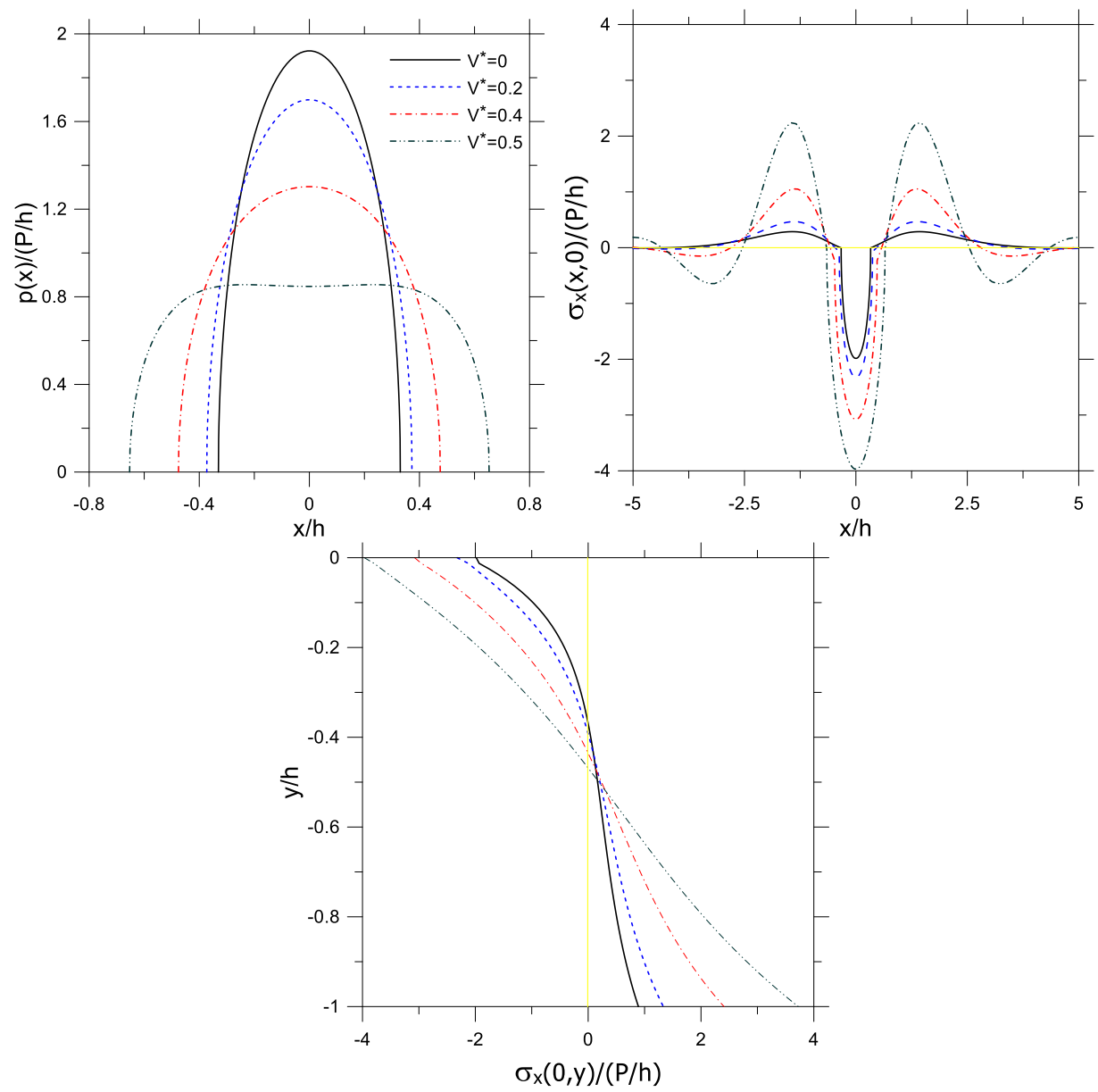

Fig 4. Contact stress and in-plane stress distributions for different values of moving velocity $V^{*}=V^{2} \rho / \mu$. $\left(R / h=100, \mu /(P / h)=500, k_{w} / \mu=1\right)$ 
Fig 5. shows the effect of the stiffness of the Winkler foundation on the contact stress and the inplane stresses. An increasing value of the stiffness of the elastic foundation tend to increase the peak values of the contact stress. As the elastic foundation is more flexible $\left(k_{w}=0.1\right)$ the axial stress $\sigma_{x}(0, y) /(P / h)$ distribution becomes linear and it matches the simple supported beam stress distribution. Also, the bigger tensile peaks occur when the foundation is softer, and the layer become susceptible to a possible cracking problem.

The effect of punch radius on the contact stress and the in-plane stresses is depicted in Fig 6. With the increasing values of the punch radius the peak values of contact stress and in-plane stresses decrease. The peak values occur when the punch radius $R / h=10$. This is because when the punch radius smaller the loading approaches to the point load case.
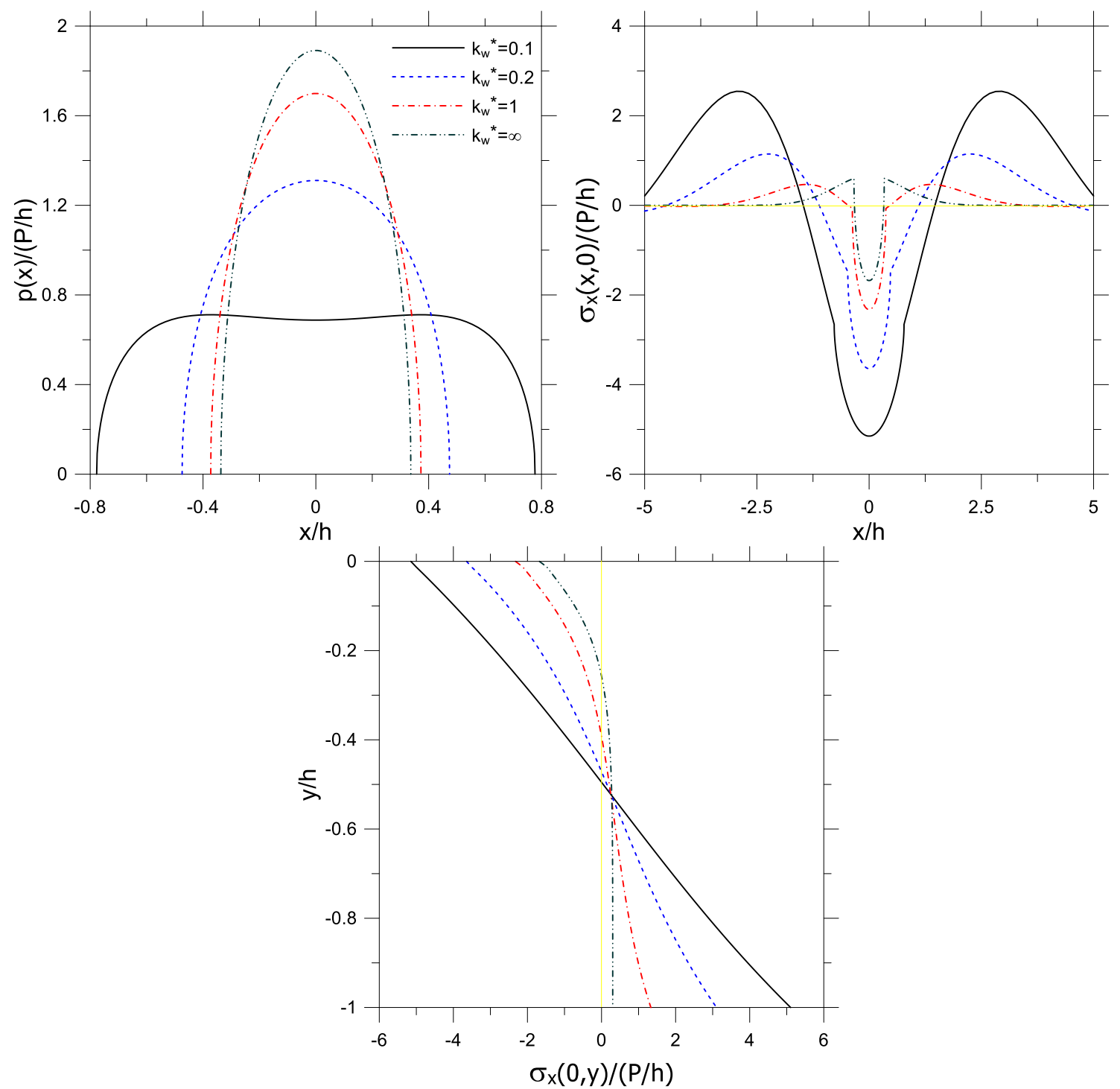

Fig 5. Contact stress and in-plane stress distributions for different values of the stiffness of Winkler foundation $k_{w}^{*}=k_{w} / \mu .\left(V^{2} \rho / \mu=0.2, R / h=100, \mu /(P / h)=500\right)$ 

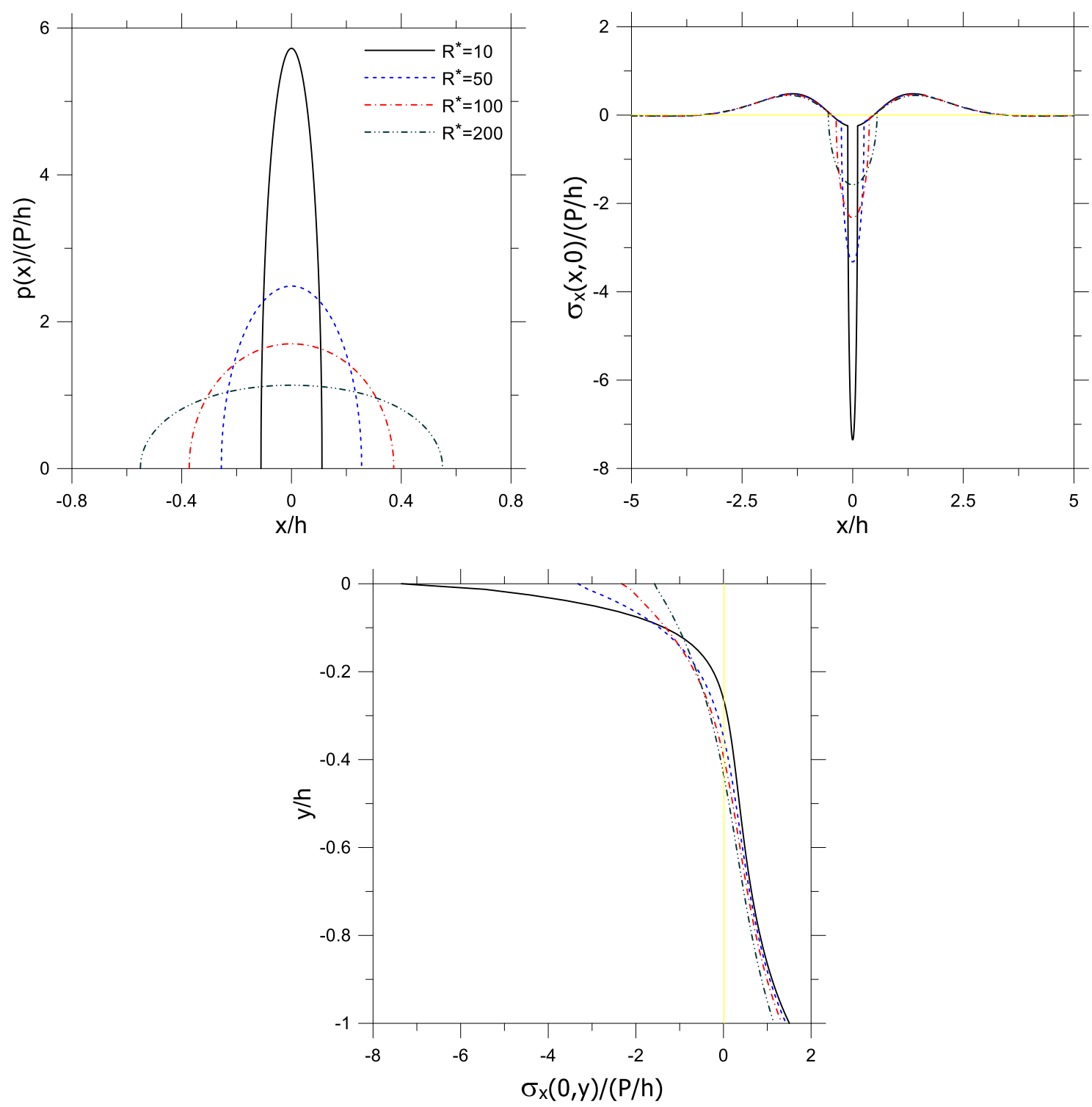

Fig 6. Contact stress and in-plane stress distributions for different values of the punch radius $R^{*}=R / h$. $\left(V^{2} \rho / \mu=0.2, k_{w} / \mu=1, \mu /(P / h)=500\right)$

\section{Conclusion}

This study is concerned with the frictionless moving contact problem between the rigid cylindrical punch and a homogeneous layer lying on a Winkler foundation. The plane contact problem is solved in the framework of linear elasticity and using Fourier integral transform technique. The effect of the moving velocity, the stiffness of the Winkler foundation, punch radius and external load on the contact area and distributions of the stress components are given.
The contact width tends to widen when the punch moves faster and the Winkler foundation getting flexible. The contact width also increases with increasing values of external load and punch radius. The contact stress increases with the increasing stiffness of the Winkler foundation and decreasing values of moving velocity. The in-plane stress distributions imply that the tensile peaks occur at the bottom and on the surface of the layer which should be considered for crack propagation problem. The tensile stress tends to increase when 
the punch moves faster, and the Winkler foundation becomes flexible.

\section{References}

[1] Guler MA, Erdogan F (2004) Contact mechanics of graded coatings. International Journal of Solids and Structures 41(14): 3865-3889.

[2] Guler MA, Erdogan F (2007) The frictional sliding contact problems of rigid parabolic and cylindrical stamps on graded coatings. International Journal of Mechanical Sciences 49(2): 161-182.

[3] Alinia Y, Beheshti A, Guler MA, El-Borgi S, Polycarpou AA (2016) Sliding contact analysis of functionally graded coating/substrate system. Mechanics of Materials 94: 142-155.

[4] Ke LL, Wang YS (2007) Two-dimensional sliding frictional contact of functionally graded materials. European Journal of Mechanics-A/Solids 26(1): 171-188.

[5] Chen P, Chen S (2012) Contact behaviors of a rigid punch and a homogeneous half-space coated with a graded layer. Acta Mechanica 223(3): 563-577.

[6] Choi, HJ (2009) On the plane contact problem of a functionally graded elastic layer loaded by a frictional sliding flat punch. Journal of Mechanical Science and Technology 23(10): 2703-2713.

[7] Chen P, Chen S, Peng J (2015) Frictional contact of a rigid punch on an arbitrarily oriented gradient half-plane. Acta Mechanica 226(12): 4207-4221.

[8] Dag S, Guler MA, Yildirim B, Ozatag AC (2009) Sliding frictional contact between a rigid punch and a laterally graded elastic medium. International Journal of Solids and Structures 46(22): 40384053.

[9] Dag S (2016). Consideration of spatial variation of the friction coefficient in contact mechanics analysis of laterally graded materials. ZAMMJournal of Applied Mathematics and Mechanics 96(1): 121-136.

[10] Liu J, Ke LL, Wang YS (2011) Two-dimensional thermoelastic contact problem of functionally graded materials involving frictional heating. International Journal of Solids and Structures 48(18): 2536-2548.

[11] Choi HJ, Paulino GH (2008) Thermoelastic contact mechanics for a flat punch sliding over a graded coating/substrate system with frictional heat generation. Journal of the Mechanics and Physics of Solids 56(4): 1673-1692.
[12] Balci MN, Dag S, Yildirim B (2017). Subsurface stresses in graded coatings subjected to frictional contact with heat generation. Journal of Thermal Stresses 40(4): 517-534.

[13] Barik SP, Kanoria M, Chaudhuri PK (2008) Steady state thermoelastic contact problem in a functionally graded material. International Journal of Engineering Science 46(8): 775-789.

[14] Mao JJ, Ke LL, Wang YS (2015). Thermoelastic instability of a functionally graded layer interacting with a homogeneous layer. International Journal of Mechanical Sciences 99: 218-227.

[15] Rhimi M, El-Borgi S, Said WB, Jemaa FB (2009) A receding contact axisymmetric problem between a functionally graded layer and a homogeneous substrate. International Journal of Solids and Structures 46(20): 3633-3642.

[16] Rhimi M, El-Borgi S, Lajnef N (2011) A double receding contact axisymmetric problem between a functionally graded layer and a homogeneous substrate. Mechanics of Materials 43(12): 787-798.

[17] El-Borgi S, Usman S, Güler MA (2014). A frictional receding contact plane problem between a functionally graded layer and a homogeneous substrate. International Journal of Solids and Structures 51(25): 4462-4476.

[18] El-Borgi S, Çömez I (2017) A receding frictional contact problem between a graded layer and a homogeneous substrate pressed by a rigid punch. Mechanics of Materials 114: 201-214.

[19] Comez I, Birinci A, Erdol R (2004) Double receding contact problem for a rigid stamp and two elastic layers. European Journal of MechanicsA/Solids 23(2): 301-309.

[20] Kahya V, Ozsahin TS, Birinci A, Erdol R (2007) A receding contact problem for an anisotropic elastic medium consisting of a layer and a half plane. International Journal of Solids and Structures, 44(17): 5695-5710.

[21] Adibelli H, Comez I, Erdol R (2013) Receding contact problem for a coated layer and a half-plane loaded by a rigid cylindrical stamp. Archives of Mechanics 65(3): 219-236.

[22] Çömez İ. (2010). Frictional contact problem for a rigid cylindrical stamp and an elastic layer resting on a half plane. International Journal of Solids and Structures 47(7): 1090-1097.

[23] Parel KS., Hills DA (2016) Frictional receding contact analysis of a layer on a half-plane subjected to semi-infinite surface pressure. International Journal of Mechanical Sciences 108: 137-143. 
[24] Yan J, Li X (2015) Double receding contact plane problem between a functionally graded layer and an elastic layer. European Journal of MechanicsA/Solids 53: 143-150.

[25] Yan J, Mi C (2017) On the receding contact between an inhomogeneously coated elastic layer and a homogeneous half-plane. Mechanics of Materials 112: 18-27.

[26] Çömez İ (2013) Contact problem of a functionally graded layer resting on a Winkler foundation. Acta Mechanica 224(11): 28-33.

[27] Dempsey JP, Zhao ZG, Minnetyan L, Li H (1990) Plane contact of an elastic layer supported by a Winkler foundation. Journal of Applied Mechanics 57(4): 974-980.

[28] Dempsey JP, Zhao ZG, Li H (1991) Axisymmetric indentation of an elastic layer supported by a Winkler foundation. International Journal of Solids and Structures 27(1): 73-87.

[29] Woźniak M, Hummel A, Pauk VJ (2002) Axisymmetric contact problems for an elastic layer resting on a rigid base with a Winkler type excavitation. International Journal of Solids and Structures 39(15): 4117-4131.

[30] Birinci A, Çakıroğlu M, Erdöl R (2002) Two Strips Problem Resting on an Elastic Foundation. Mathematical and Computational Applications 7(3): 275-286.

[31] Birinci A, Adıyaman G, Yaylacı M, Öner E (2015) Analysis of continuous and discontinuous cases of a contact problem using analytical method and FEM. Latin American Journal of Solids and Structures 12(9): 1771-1789.

[32] Adiyaman G, Birinci A (2018) A general solution for the receding contact problem of a functionally graded layer resting on a Winkler foundation. Journal of Structural Engineering \& Applied Mechanics 1 (3): 136-146.

[33] Galin LA. Contact problems: the legacy of L.A. Galin. Ed. G.M.L. Gladwell (Ed.) Springer, Dordrecht, 2008.
[34] Georgiadis HG (1987) Moving punch on a highly orthotropic elastic layer. Acta Mechanica 68(3): 193-202.

[35] De J, Patra B (1994) Dynamic punch problems in an orthotropic elastic half-plane. Indian Journal of Pure and Applied Mathematics, 25: 767-767.

[36] Zhou YT, Lee KY (2012a) Contact problem for magneto-electro-elastic half-plane materials indented by a moving punch. Part I: closed-form solutions. International Journal of Solids and Structures 49(26): 3853-3865.

[37] Zhou YT, Lee KY (2012b) Contact problem for magneto-electro-elastic half-plane materials indented by a moving punch. Part II: numerical results. International Journal of Solids and Structures, 49(26), 3866-3882.

[38] Zhou YT, Lee KY, Jang YH (2013) Influences of the moving velocity and material property on frictionless contact problem of orthotropic materials indented by a moving punch. Archives of Mechanics 65(3): 195-217.

[39] Zhou YT, Lee KY, Jang YH (2014) Indentation theory on orthotropic materials subjected to a frictional moving punch. Archives of Mechanics 66(2): 71-94.

[40] Çömez İ (2015) Contact problem for a functionally graded layer indented by a moving punch. International Journal of Mechanical Sciences 100: 339-344.

[41] Çömez İ (2017) Frictional moving contact problem for a layer indented by a rigid cylindrical punch. Archive of Applied Mechanics, 87(12): 19932002.

[42] Balci MN, Dag S (2018) Dynamic frictional contact problems involving elastic coatings. Tribology International 124: 70-92.

[43] Erdogan F. Mixed boundary value problems in mechanics. In: Nemat-Nasser S, editor. Mech Today, 4. Oxford: Pergamon Press, 1978.

[44] Krenk S (1975) On the use of the interpolation polynomial for solutions of singular integral equations. Quarterly of Applied Mathematics 32: 479-484.

\section{Appendix A}

Expressions of $k_{1}(x, \xi)$ and $\phi_{1}$ appearing in (14) are given below:

$$
k_{1}(x, \xi)=\frac{1}{\phi_{1}} \int_{0}^{\infty} M_{1}(\alpha) \sin \alpha(\xi-x) d \alpha
$$




$$
\begin{aligned}
M_{1}(\alpha)= & -\left(\left(-1+n_{1}^{2}\right) n_{2}\left(1+n_{1}^{2}\right)^{2}\left(-1+e^{2 n_{1} h \alpha}\right)\left(1+e^{2 n_{2} h \alpha}\right) \alpha\right. \\
& +n_{2}\left(-1+e^{2 n_{2} h \alpha}\right)\left(\left(-1+n_{1}^{2}\right)\left(-1+e^{2 n_{1} h \alpha}\right) k_{w} / \mu\right. \\
& \left.\left.\left.-4 n_{1}\left(1+e^{2 n_{1} h \alpha}\right) \alpha\right)\right)\right) / \Delta \\
\Delta= & \left(\left(1+n_{1}^{2}\right)^{4}\left(-1+e^{2 n_{1} h \alpha}\right)\left(-1+e^{2 n_{2} h \alpha}\right) \alpha\right. \\
- & 4 n_{1} n_{2}^{2}\left(-1+e^{2 n_{2} h \alpha}\right)\left(\left(-1+n_{1}^{2}\right)\left(1+e^{2 n_{1} h \alpha}\right) k_{w} / \mu\right. \\
- & \left.4 n_{1}\left(-1+e^{2 n_{1} h \alpha}\right) \alpha\right) \\
+ & \left(1+n_{1}^{2}\right)^{2} n_{2}\left(\left(-1+n_{1}^{2}\right)\left(-1+e^{2 n_{1} h \alpha}\right)\left(1+e^{2 n_{2} h \alpha}\right) k_{w} / \mu\right. \\
- & \left.\left.\left.8 n_{1}\left(1+e^{2 n_{1} h \alpha}+e^{2 n_{2} h \alpha}-4 e^{\left(n_{1}+n_{2}\right) h \alpha}+e^{2\left(n_{1}+n_{2}\right) h \alpha}\right) \alpha\right)\right)\right)
\end{aligned}
$$

$\varphi_{1}=\lim _{\alpha \rightarrow \infty} M_{1}(\alpha)$ 